\title{
Thermographycal Analysis of the coking oil-products degree
}

\author{
Vladimir Zaharenko
}

Rendering and graphic analysis of the infrared fields on the technological equipment surfaces actualize new informing possibilities for the purpose of inspecting the technological processes. The high thermographic control informativeness is connected first of all with the rendition of energetical transformation in the control objects, as far as the energy transformation process is in most cases connected with release or absorbency of the warm and these alternations rendering makes it possible to follow the very energetical transformation's dynamics.

The work at the termographic control systems, adapted for the purposes of the ceaseless termographic inspecting of different technological processes in the conditions of the main technological equipment's exploitation is carried out in Omsk State Technical University since 1993, and especially for the purpose the inspecting of the temperature fields of whirling furnaces producting cement, haydite, oil-coke, lime burning, bake-out in aluminum industry.

The main demands for such system are conditions of temperature fields control in the range $(100-600)^{0} \mathrm{C}$ with absolute accuracy $(3-5)^{0} \mathrm{C}$, working ability while medium thermal change from $-40{ }^{\circ} \mathrm{C}$ to $+60{ }^{\circ} \mathrm{C}$, high operation reliability, providing continuous work of the system during some years, solving the problem of measurement assurance.

In this work, the coking degree control is supposed to be indirect, according to the temperature gradient along vertical forming external surface of the coke cell. The statement of the connection of the temperature gradient $\triangle T$ on the reactor surface with the phasic conversion dynamics inside the reactor is based on the following notions of the coking processes. The fresh breeze, warmed to $470 . .490{ }^{0} \mathrm{C}$, chuted through the inferior reactor neck, fills the space above the formed coke. At certain temperature and pressure, the coking processes proceed with fluid products releasing. At reactions' process coke takes the inferior part of the reactor, and fluid takes the superior, above the liquid layer. The thermal transmission density between the 
http://dx.doi.org/10.21611/qirt.2006.071

reactor's panel and products, being in fluid, liquid, and solid stages, will be different. The most intensive thermal transmission will be on the liquid layer degree, the lest in the zone of fluid and solid phases. Besides, as far as the transit from liquid to fluid phase has endothermal character, there appear conditions for gradient temperature increase. At the same time, reactions of condensation, compression, and coking take place with thermal release, that also promotes increase of the temperature differential on the liquid and coke border. So, the derivatives $-\triangle T / \triangle h(h-$ coordinate of the reactor's height) above the liquid layer and under it will have different signs. Negative $-\triangle T / \triangle h$ above the liquid layer, and with the sign "plus" on the liquid and solid phases border.

The functional scheme of the coking degree control is attached.

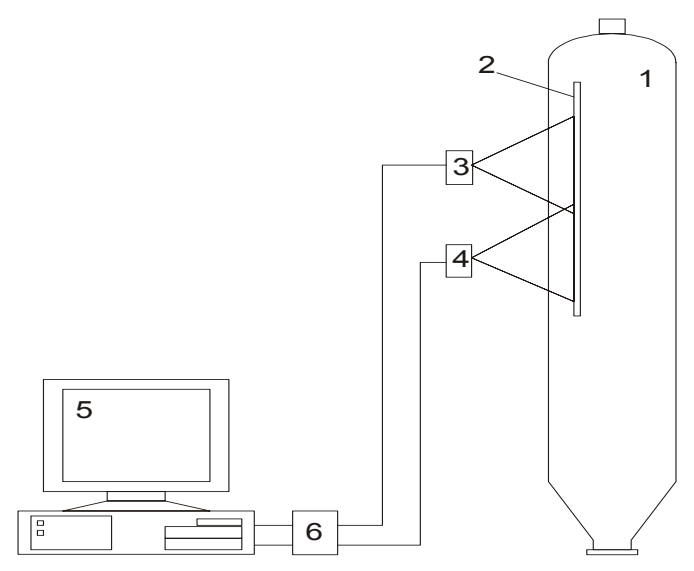

The system represents an appliance, consisting of one or several scanning pyrometric transducing apparatus 3 and 4, IBM-computer 5, channel adapter 6, programming provider refinement and visualization of the temperature fields. In the thermal insulation layer of the reactor 1 there is a $0,1 \mathrm{~m}$ wide shelter 2 . 